Peer Reviewed Review openaccess

\title{
Raman imaging of protein in a model cheese system
}

\author{
Elizabeth Nickless* and Stephen E. Holroyd \\ Fonterra Research and Development Centre, Private Bag 11 029, Palmerston North 4442, New Zealand \\ Contact \\ Elizabeth Nickless (elizabeth.nickless@fonterra.com)
}

\begin{abstract}
Raman confocal microscopy is an increasingly useful technique when applied to food samples; it has the unique ability to interrogate the chemical structure, aligned with the same confocality capability that is available when using standard confocal microscopy. In this research, we investigated the potential of Raman confocal microscopy to investigate the components in a model cheese system. We showed an ability to distinguish whey protein particles within the casein protein matrix using several different image analysis approaches. The results illustrate the potential of Raman confocal microscopy and imaging to understand the chemical and microstructural features of cheese systems via the analysis of the distribution of the protein types in complex dairy matrices.
\end{abstract}

Keywords: Raman, imaging, cheese, protein, confocal, microscopy, casein, whey

\section{Introduction}

The potential to apply analytical spectroscopic methods to analyse the microstructure of food systems has been a relatively recent development, largely because of the increasing capability and availability of suitable imaging technologies. Developments in instrumentation and spectral and spatial resolution and increased signal-to-noise technologies allow detailed chemical and structural information to be gathered at a microscale in food systems. ${ }^{1}$

Dairy systems, in particular, present many challenges with respect to microstructural imaging; the limited specific histological techniques that are available can introduce artefacts. The development of Raman imaging using confocal microscope systems allows research into the chemical and corresponding microstructure of various components in a food system without some of the many artefacts introduced with other methods. ${ }^{2}$

Protein secondary and tertiary structure is linked to functionality in many food systems and plays an important role in the development of new food products. During the processing of food products, changes, such as heating and shear, that alter the protein structure and component interactions and hence the functionality may occur. Raman spectroscopy and imaging has been shown to be a versatile tool for investigating the chemistry and structure of a range of food systems including dairy products. $^{3-6}$ In the case of dairy proteins, their Raman spectra can be interrogated to monitor changes in the secondary structure, sulphydryl groups, carboxylic acid groups and
Correspondence

E. Nickless (elizabeth.nickless@fonterra.com)

Received: 14 June 2020

Revised: 14 August 2020

Accepted: 17 August 2020

Publication: 20 August 2020

doi: 10.1255/jsi.2020.a9

ISSN: $2040-4565$

\section{Citation \\ E. Nickless and S.E. Holroyd, "Raman imaging of protein in a model cheese system", J. Spectral Imaging 9, a9 (2020). https://doi.org/10.1255/jsi.2020.a9 \\ (c) 2020 The Authors}

This licence permits you to use, share, copy and redistribute the paper in any medium or any format provided that a full citation to the original paper in this journal is given. 
the micro-environment of aromatic side chains. ${ }^{7,8}$ Raman spectroscopy has the potential to unlock information that relates changes in the chemical structure and distribution of food components to the functionality in food systems. $^{8-10}$

The application of spectral methods to investigate the chemical structure within various dairy food systems is very useful for analysing the effects of processing and composition on individual food component systems. In addition, imaging techniques using Raman spectroscopy allow the identification and location of various structures within a food sample at a microscale; this enhances the capability of Raman spectroscopy to elucidate structurefunction relationships in food systems.

Furthermore, Raman spectroscopy offers the potential to elucidate more detailed information from specific protein components in a food system. Protein and peptide structures contain amide bonds that give Raman signals at specific frequencies, particularly in the amide I $\left(1600-1700 \mathrm{~cm}^{-1}\right)$ and amide III $\left(1235-1270 \mathrm{~cm}^{-1}\right)$ regions. The exact frequencies of the amide bands depend on the relative positions of protein side chains and interactions between other chemical groups, i.e. of the $\mathrm{C}=\mathrm{O}, \mathrm{C}-\mathrm{N}$ and $\mathrm{N}-\mathrm{H}$ groups. Protein secondary struture is characterised by $a$-helices, $\beta$-sheets and disordered structures. Protein tertiary structure is dependent on the side chains of the amino acid chains and their interactions; these are generally hydrogen bonding, hydrophobic interactions and disulphide bridges. ${ }^{5,11-13}$ These structures give rise to a unique spectral fingerprint that is due to both the amide backbone and the side chains. This fingerprint is the key element in the application of Raman spectroscopy in the determination of protein structure. The purpose of this study was to distinguish different regions of protein in the Raman images of model cheese systems by using different chemometric techniques to compare the spectral data from each pixel and then to group them into clusters.

\section{Materials and methods}

Raman imaging was performed on a WITec Raman confocal microscope with a Princeton Instruments Acton SP2300 spectrograph, a $0.3 \mathrm{~m}$ imaging triple grating monochromator, WITec Control Software Version 1.60.3.3 and a $532 \mathrm{~nm}$ Raman excitation laser, the Spectra-Physics Excelsior 532-60. Samples were scanned at an excitation wavelength of $532 \mathrm{~nm}$ with the T1: $600 \mathrm{gr} \mathrm{mm}^{-1}$
$\mathrm{BLZ}=500 \mathrm{~nm}$ grating. This gave a spectral resolution of $2-3 \mathrm{~cm}^{-1}$. The model cheese system was prepared at laboratory scale and whey protein particles were added during the final blending.

The cheese samples were subsampled with 1-2g quantities and then sectioned on a Leica cryostome CM3050 S at $-20^{\circ} \mathrm{C}$ to allow sections that retained the microstructure of the sample to be obtained. Images of the sample were then immediately scanned at $4^{\circ} \mathrm{C}$ using a thermoelectric heating and cooling stage (PE120 Linkam) with a $100 \times$ NA 1.4 Zeiss oil immersion lens. On this instrument, each image scan took approximately $1.5 \mathrm{~h}$.

Images were taken at 10-15 $\mu \mathrm{m}$ below the cut surface of the sample and random positions in each sample were scanned.

Each image was scanned at 200 points and 200 lines per $50 \mu \mathrm{m}^{2}$ area with excitation at $532 \mathrm{~nm}$, an integration time of $0.15 \mathrm{~s}$ and a laser power of $15 \mathrm{~mW}(40,000$ individual spectra per image). The laser power was set at $15 \mathrm{~mW}$ as initial tests had shown that laser powers above $20 \mathrm{~mW}$ could lead to degradation of the sample, although this does depend on the scan temperature when using a controlled-temperature stage; cooling at $4{ }^{\circ} \mathrm{C}$ can allow slightly higher laser powers of up to $20 \mathrm{~mW}$ to be used for image scanning of this sample type.

Due to the number of spectra generated for each image, multivariate methods of imaging are a very effective way to image a range of chemical structures in an image using spectral fingerprint data. In this case, Raman spectral data with a number of wavelengths within the spectral range $350-3600 \mathrm{~cm}^{-1}$ were used for analysis; as the reported "fingerprint" region of approximately $350-1800 \mathrm{~cm}^{-1}$ removed the differentiation of some components of interest in dairy components, the broad $\mathrm{C}-\mathrm{H}$ and $\mathrm{O}-\mathrm{H}$ regions were included in our analysis with these methods.

Two main different sample types were used: a control sample of a standard cheese and the same cheese with whey protein particles added. In addition, a control sample of the whey particles was analysed to establish a spectral and microstructural reference for the whey particles. The image data were then processed using WITec Project 5.1 with background correction, cosmic spike removal and Savitsky-Golay smoothing applied. In order to contrast differing image analysis approaches with images of complex heterogeneous foods, such as cheese, the images were mapped out using several approaches; integrated peak maps of a range of chemical bonds and a range 
of unsupervised clustering algorithms; K-means clustering, multivariate curve resolution with unconstrained alternating least squares (MCR-ALS) and non-negative matrix factorisation (NMF); these methods are available as options within the WITec software Project 5.1. The additional aim was to test the capability of the software applications available to us to reconstruct accurate images of the cheese sample along with relevant spectral information. Unsupervised methods require the user to enter endmember numbers. In this experiment endmember numbers were chosen for the non-supervised algorithms based on the known number of main ingredients, namely fat, protein and the whey particles, and were increased by one each repetition until the whey casein particles were visible in the resulting image.

Integrated peak maps enable the analysis of the distribution and concentrations of various chemical bonds pertaining to fat, protein and other cheese ingredients in an image. This is a useful way to identify and interrogate chemical components, especially if they contain a unique bond type or a predominant bond type in their chemical structure, and it also allows an interpretation of the distribution of specific bonds within the microsctructure of a sample. $^{14}$

In addition, chemical components that share chemical bond types can often be differentiated based on the ratio of two relevant bonds in any one compound, and very similar compounds may be differentiated using multivariate methods, such as principal components analysis or $\mathrm{K}$-means clustering, that separate compounds according to the whole or part of their spectral fingerprint. ${ }^{15}$

\section{Imaging using peak interval maps}

Peak interval maps were generated from the integrated spectral intensity around a specified peak position using the average binomial distribution and a bandwidth of $5 \mathrm{~cm}^{-1}$. The binomial function averages the spectral pixel window using binomial-distributed weighting factors, with the maps generated showing the distribution and concentration of the specified component peak representing a specific type of chemical bond, effectively a chemical map. If any one constituent of a sample has a unique chemical bond in comparison with other constituents in the sample, a resultant distribution map of that specific component is obtained.

For peak interval maps, relevant peak positions were chosen from the available reference literature (see Table 1) and spectral regions that showed the best

Table 1. Raman peaks of interest that were evaluated using band widths reported for dairy proteins and fats.

\begin{tabular}{|l|l|}
\hline Band assignment & Wavenumber $\left(\mathrm{cm}^{-1}\right)$ \\
\hline S-S stretch Cysteine & $510,525,545$ \\
\hline S-H stretch Cysteine & $2550-2580$ \\
\hline C-S stretch Cysteine, Methionine & $630-670,700-745$ \\
\hline Tryptophan & $760,880,1360$ \\
\hline Tyrosine doublet & $850 / 830$ \\
\hline Phenylalanine & 1005 \\
\hline C=O stretch of COO-, COOH & 1400,1730 \\
\hline C-H bend & 1453 \\
\hline- C-H or $+\mathrm{C}-\mathrm{H}$ stretch & $2880,2930,3060$ \\
\hline a-Helix & 938 \\
\hline Amide I & $1650-1685$ \\
\hline Amide III & $1235-1270$ \\
\hline Aromatic side chains (Phe, Tryp, Tyr) & $3060-3100$ \\
\hline Acyl chains fatty acids & 2850,2885 \\
\hline C=O carbonyl fats & 1744 \\
\hline O-H stretch & 3200 \\
\hline
\end{tabular}


differentiation of the components in the cheese sample were selected. Peaks relating to several protein structures of interest were investigated to allow an understanding of the distribution of protein conformational structures, concentrating on those that would differentiate protein structures between the main casein matrix of the model cheese and the whey protein particles that were added to the cheese matrix. Figure 1 illustrates the type of spectral information that can be obtained from using a peak interval map of the casein region in the image of the model cheese system.

The amide I band is due mainly to $\mathrm{C}-\mathrm{O}$ and $\mathrm{N}-\mathrm{H}$ bond stretches, whereas the amide $\mathrm{II}$ band is a mixture of $\mathrm{C}-\mathrm{N}$ stretching and $\mathrm{H}-\mathrm{N}-\mathrm{C}$ bending. Differences in these bands can reflect the relative differences in whey protein and casein secondary structures and thus distributions. Figure 2 shows how this peak interval information, gathered over the $\mathrm{CH}_{3}$ protein terminus region (2950-3000 $\left.\mathrm{cm}^{-1}\right)$, can be combined into a single image by mapping intensity distributions of the whey protein signals to provide unique information on the chemical compositional profile of the sample.

Figures 3 and 6 illustrate how peak intervals of this type for three wavelength ranges can be combined to give an image with rich chemical information on the location of both whey protein and casein peaks imaged at $1613 \mathrm{~cm}^{-1}$ and $3100 \mathrm{~cm}^{-1}$, along with fat imaged at $2880 \mathrm{~cm}^{-1}$. This approach can be further extended to look at the differences between whey protein and casein in terms of their respective amide I and amide III bands and aromatic side chain contents.

\section{Results and discussion}

Representative spectra for the whey protein and casein matrices were gathered by averaging regions of interest (ROIs) from within the two different protein matrices from the cheese image (Figure 4A). Figures $4 A$ and $B$ and Figure 5 illustrate the spectral differences between the

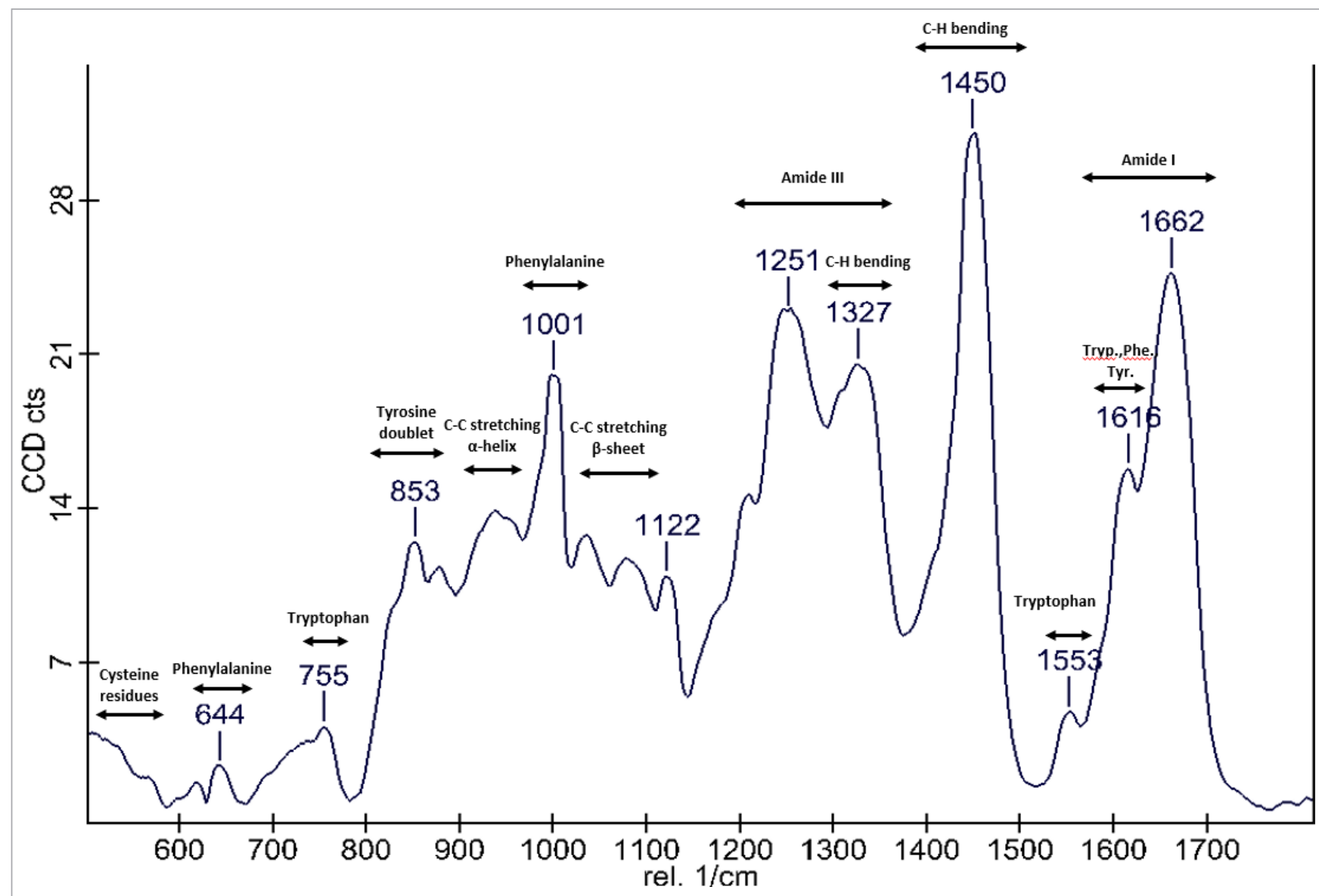

Figure 1. Spectral graph of the fingerprint region $600-1800 \mathrm{~cm}^{-1}$ of the protein matrix in the model cheese control sample. 

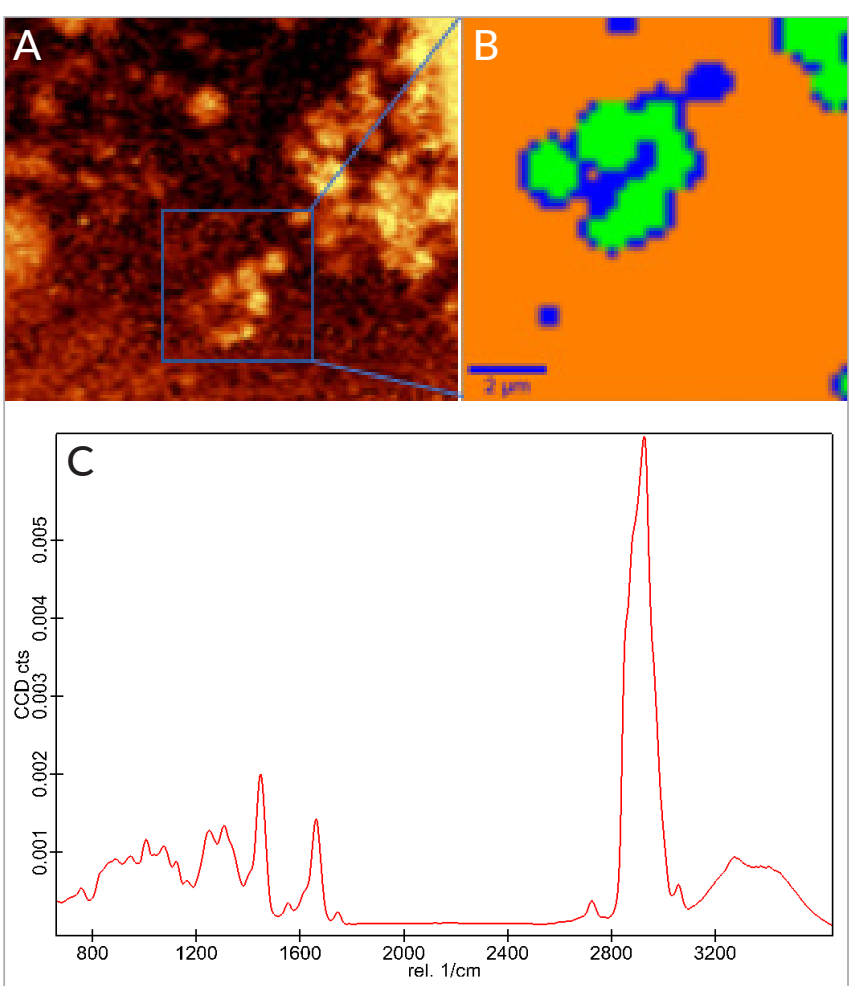

Figure 2. Raman image of whey protein particle sample. The images illustrate that the whey protein particles could be identified in the preparation solution on inspection of the spectral details from the various objects in $(A)$ the scanned image, $(B)$ the green pixels in the cluster map and $(\mathrm{C})$ their associated spectral data. casein matrix and the whey protein particles in the model cheese system, indicating differences between the two protein components. The protein matrices are able to be distinguished by reference to previous unpublished work where Raman spectra of the individual ingredients and matrices were measured along with the spectral information generated from the whey particle reference sample, as mentioned in the materials and methods section (Figure 2). The spectral data illustrate that, apart from differences across the amide regions of the Raman spectrum, the casein matrix contains relatively more aromatic side chains at $3100 \mathrm{~cm}^{-1}$. The degree of "buriedness" of the tyrosine doublet at $850 / 830 \mathrm{~cm}^{-1}$ in the spectral data shows that the relative difference in tyrosine in the casein matrix is higher than that in the whey protein particles. The amide I and amide III band distributions are also characteristically different. A slight shift in the main amide I band for the whey protein particles indicates more $\beta$-sheet structures, whereas the spectral data indicate relatively more random coil structures in the casein matrix. We suggest that Raman shifts in the amide III region indicate similar relative differences of random coil and $\beta$-sheet structures between the casein and whey protein components within the cheese system. ${ }^{12,13}$

The combined overlay of individual peak maps in Figure 6 shows good differentiation between the three

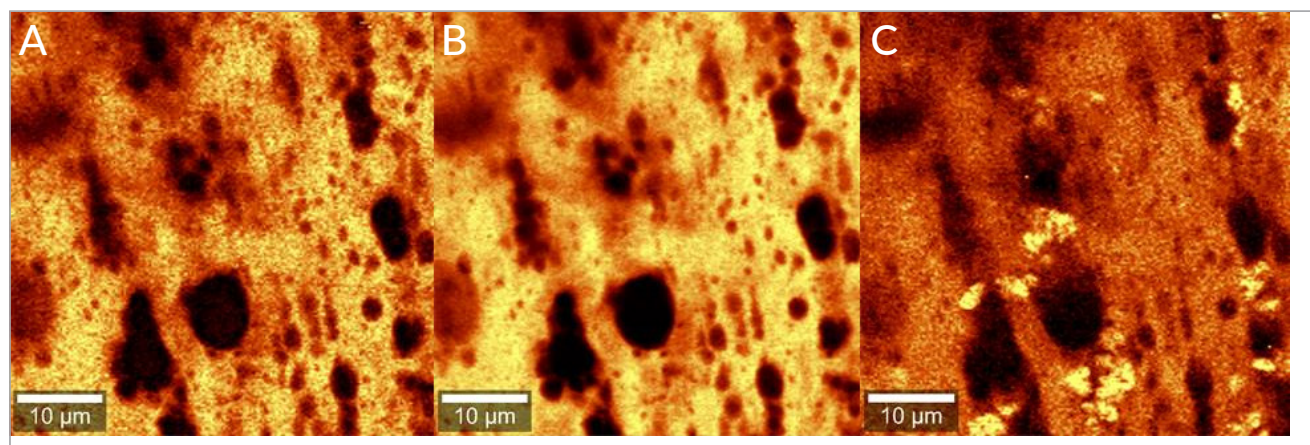

Figure 3. Peak interval maps of three different integrated intensity protein peak regions. (A) Peak interval map of the aromatic side chains $\left(3100 \mathrm{~cm}^{-1}\right)$; (B) peak interval map of the $\mathrm{O}-\mathrm{H}$ region $\left(3200 \mathrm{~cm}^{-1}\right)$; (C) peak interval map of the amide I region

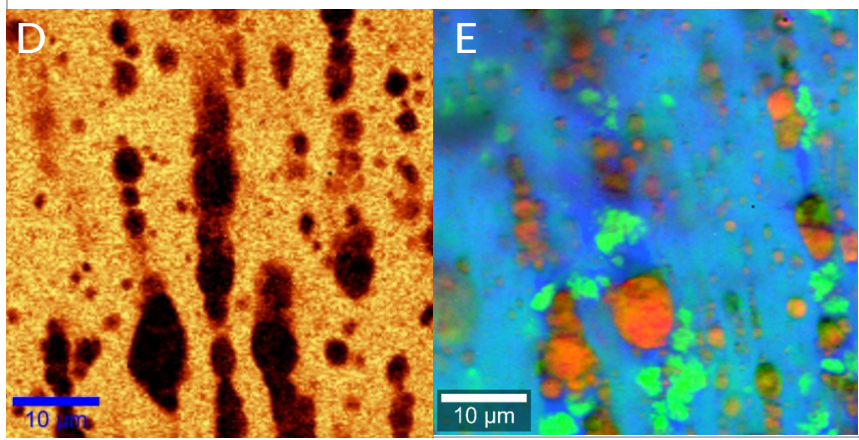
$\left(1613 \mathrm{~cm}^{-1}\right)$; (D) peak interval map of the amide I region $\left(1613 \mathrm{~cm}^{-1}\right)$ in the control sample; (E) combined overlay of three individual peak interval maps. Red = fat $\left(2880 \mathrm{~cm}^{-1}\right)$; blue = casein matrix aromatic side chains $\left(3100 \mathrm{~cm}^{-1}\right)$; green $=$ whey protein particles amide I band $\left(1613 \mathrm{~cm}^{-1}\right)$. 

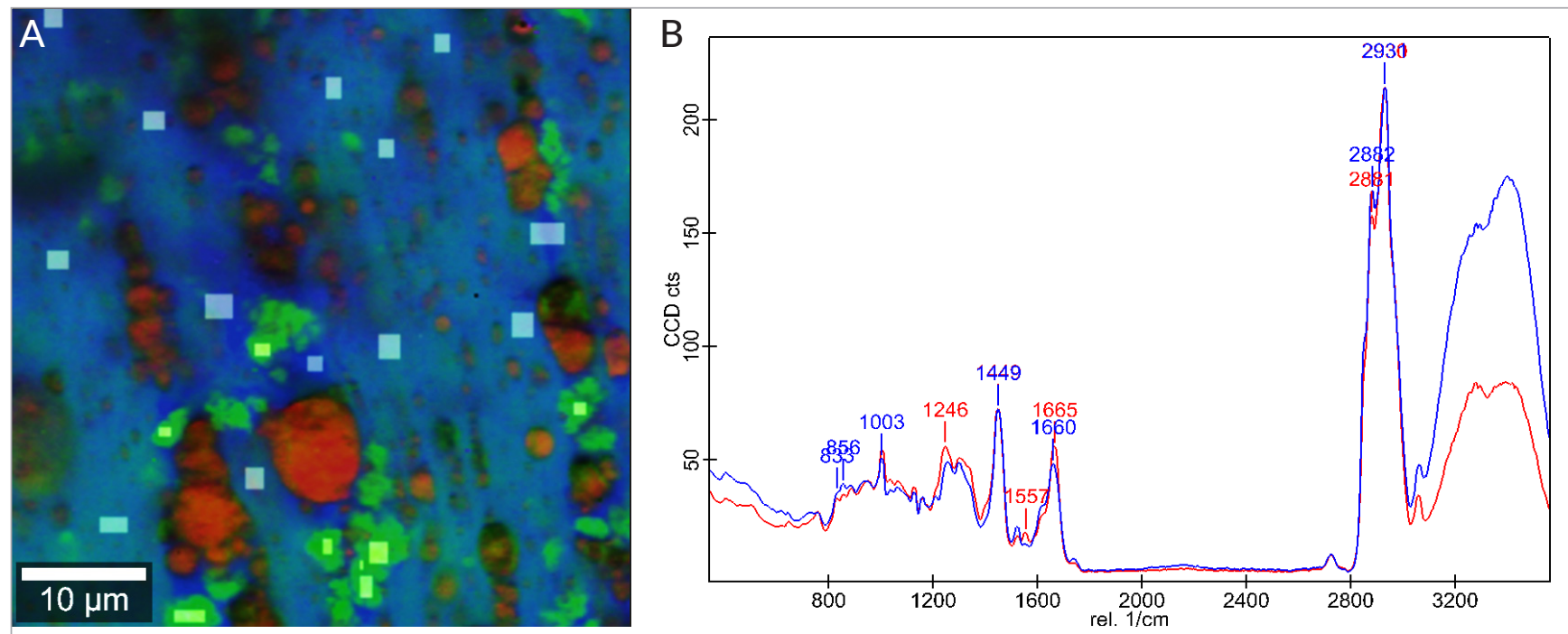

Figure 4. (A) Cheese image: the pale-blue ROls are from the cheese casein matrix and the bright-green ROls are from the whey protein particles within the cheese sample. (B) Reference spectra of the casein and whey protein components from the Raman image of the model cheese system. The blue spectrum represents the average of the casein pixel ROls and the red spectrum represents the average of all the whey protein pixel ROIs.

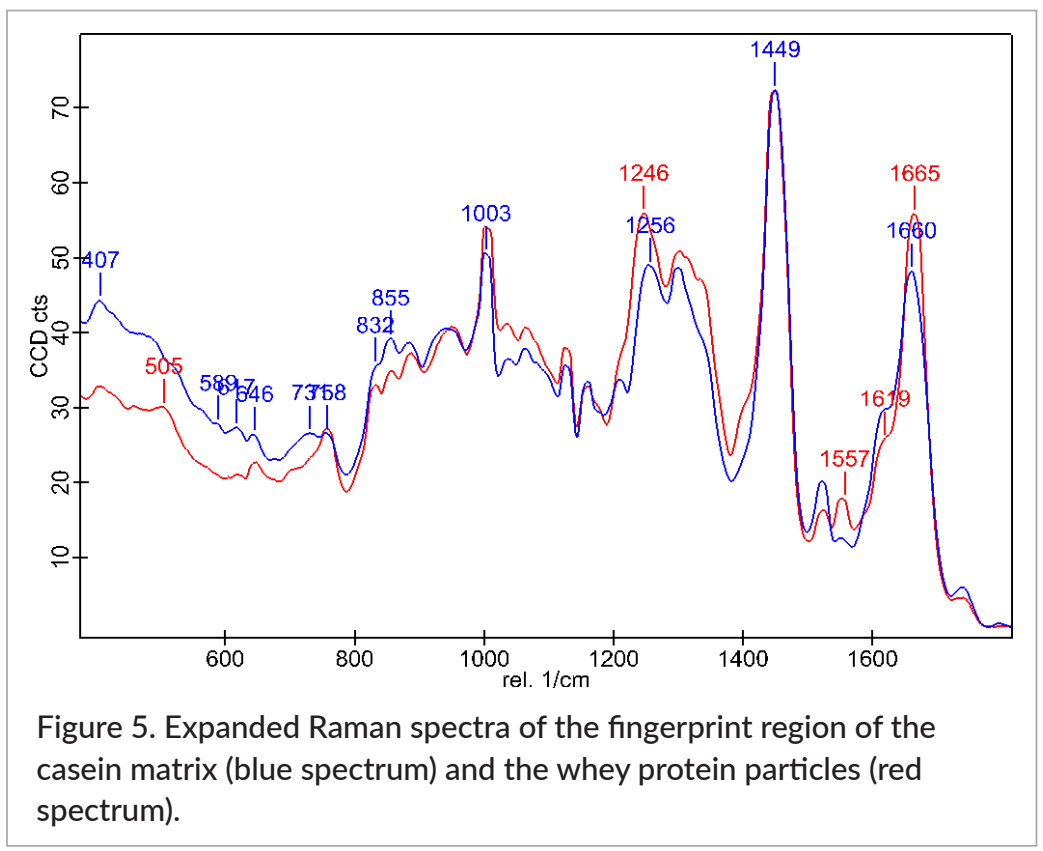

chemically distinct components: fat, whey protein and casein protein. Of particular interest is the clear ability to distinguish between whey protein and casein, the latter being an intrinsic part of the cheese structure, whereas the whey protein particles were an introduced component. They differ, with the whey protein displaying more amide III signal and greater amide I intensity, as noted previously.
The casein matrix of the cheese can also be investigated and chemical differences can be detected by detailed analysis of ROIs from the Raman spectra. Figures $4 A$ and $4 \mathrm{~B}$ and the spectra in Figure 5 show that this region is a blend of casein and water, with some significant differences in the $\mathrm{CH}_{2}\left(2845 \mathrm{~cm}^{-1}\right)$ and terminal methyl $\mathrm{CH}_{3}$ $\left(2930 \mathrm{~cm}^{-1}\right)$ regions. The amide II and amide III bands at $1522 \mathrm{~cm}^{-1}$ and $1300 \mathrm{~cm}^{-1}$ also distinguish the two forms 


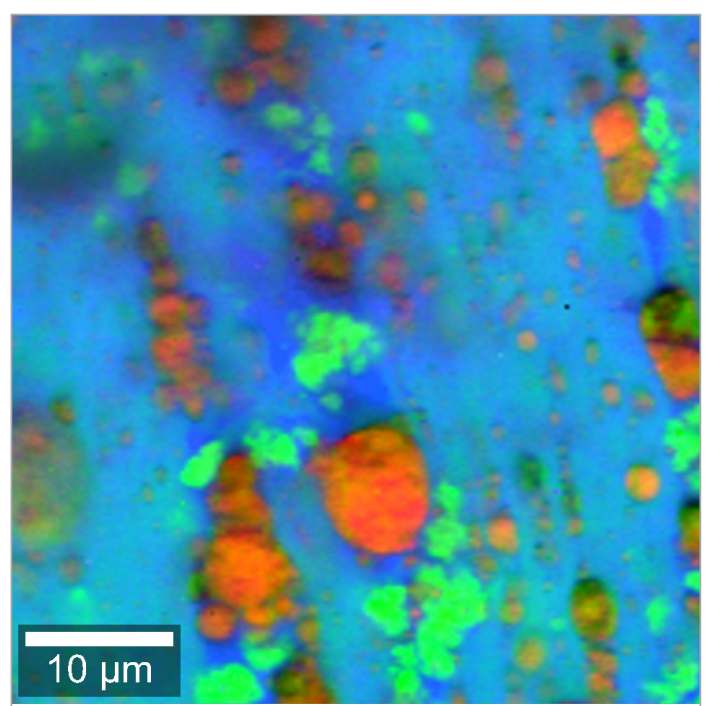

Figure 6. Combined overlay of three individual peak maps: red $=$ fat $\left(2880 \mathrm{~cm}^{-1}\right)$; blue $=$ casein matrix aromatic side chains $\left(3100 \mathrm{~cm}^{-1}\right)$; green $=$ whey protein particles amide I band $\left(1613 \mathrm{~cm}^{-1}\right)$. (We noted that the fat regions could also be readily identified by the characteristic peak at $1744 \mathrm{~cm}^{-1}$; spectra not shown here.)

of casein matrix. As casein comprises four main types of protein ( $a_{\mathrm{S}^{-}}, a_{\mathrm{S}^{-}}, \beta$ - and $\mathrm{k}$-caseins) with different secondary structures, we inferred that these were present in different concentrations in different regions in the cheese matrix.

\section{Multivariate imaging methods}

Unsupervised clustering algorithms were also used to elucidate components within the model cheese system. Unsupervised methods assume no knowledge of the number of components in a sample set and their application requires testing several variable component end member numbers to investigate results based on knowledge of the sample. In this case, although the composition of the cheese and the reference spectra of the ingredients are known, the application of a number based on knowledge of the cheese matrix or using supervised applications does not give a complete answer when there is significant interaction between the constituents. Therefore, we have found that in these examples judicious experimentation in testing component numbers with unsupervised methods is the best approach. Typically, a cheese system could be assessed to have only fat and protein (e.g. two components). However, this does not allow for the considerable heterogeneity of cheese and using three to six components gave different results in terms of distinguishing fat and protein sub-groups that appeared to differ chemically based on assessment of their Raman spectra and also based on the known cheese ingredients.

K-means clustering is a multivariate technique that groups similar spectral areas into clusters. The associated algorithm effectively maximises the distance between averaged spectral clusters and minimises the distance between samples within a cluster. In this case, the
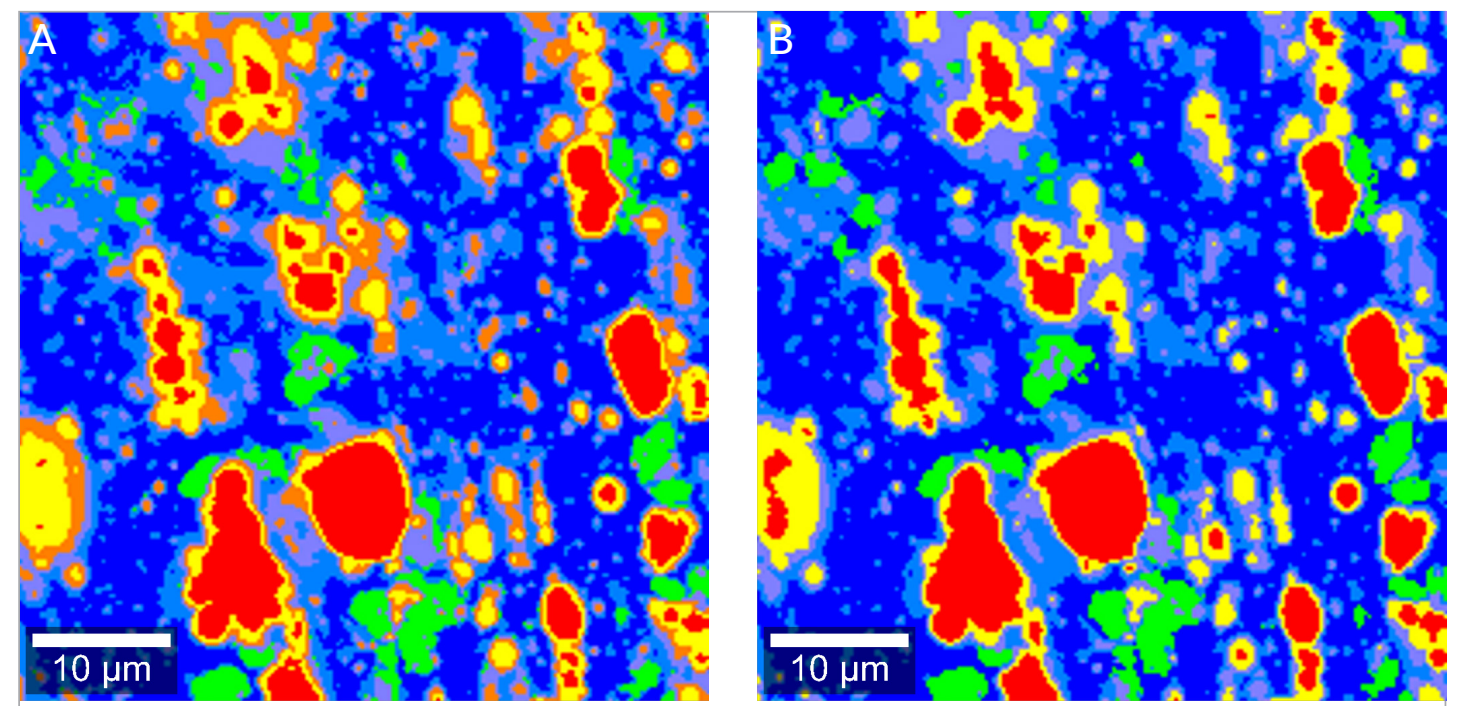

Figure 7. (A) K-means image of cheese with seven components using Manhattan distances (whey protein particles in orange). (B) K-means image of cheese with six components using Euclidean distances (whey protein particles in red). 

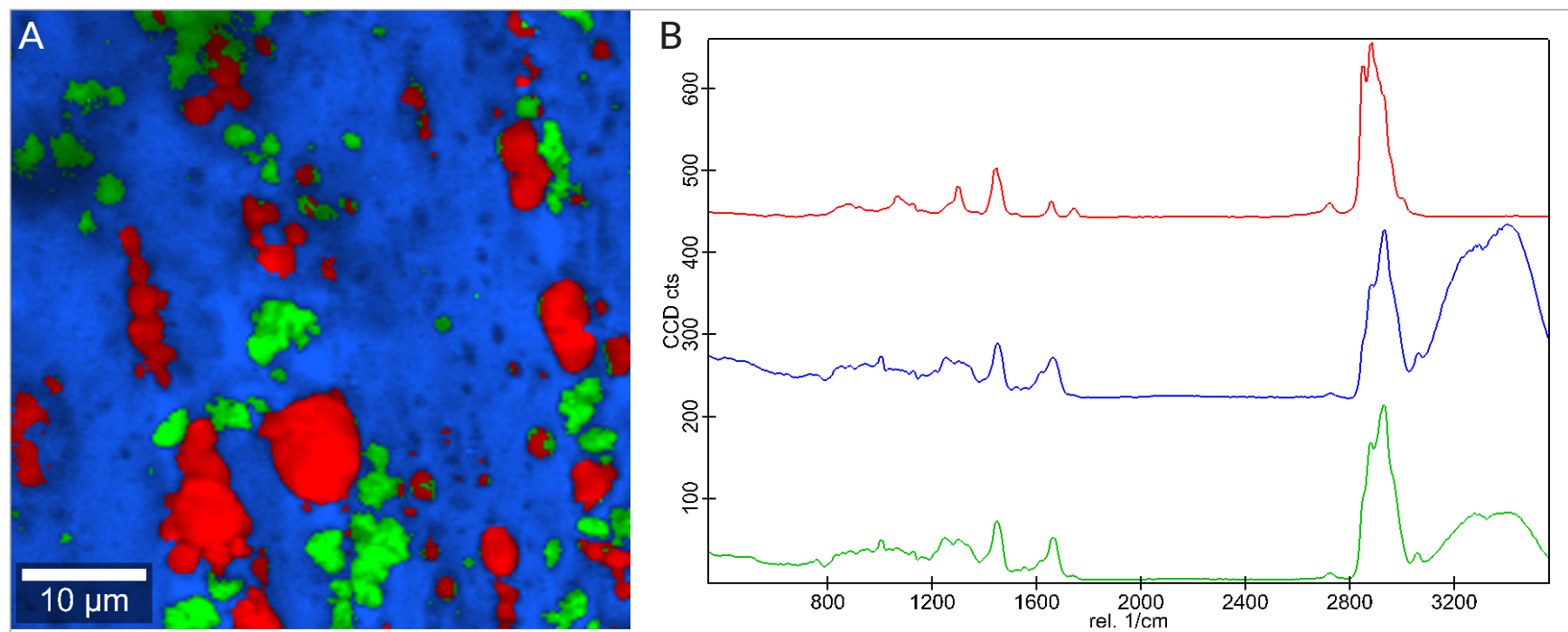

Figure 8. Cheese image and associated spectra using MCR-ALS: whey protein particles = green; fat = red; main casein matrix = blue.

individual samples were the spectra from every pixel in the image, i.e. an image of $50 \mu \mathrm{m}^{2}$ with $200 \times 200$ pixels of data gathered, giving a total of 40,000 spectra. Two techniques were applied to compare the K-means clustering: the Manhattan and Euclidean distance approaches, which differ in how they assess differences in data clusters.

The Manhattan distance application was used in Figure 7A. This captures the distance between two points by aggregating the pairwise absolute difference between each variable. The Euclidean distance application was used in Figure 7B. This captures the same information by aggregating the squared difference for each variable. Given the mathematics used, the Manhattan distance approach places less emphasis on outliers. The Manhattan approach required seven clusters to detect the whey protein particles as being separate from the other components in the image data. In contrast, using the Euclidean method on the same original image data, only six clusters were needed to detect the whey protein particles. When we compared the images from the use of these methods, we also detected certain changes in the distributions of components in other parts of the image, mainly around the interfaces between fat and protein. This reflects small but detectable differences within component types such as fat morphology (where the mobility of acyl chains varies) and also different secondary structures in proteins, expressed as changes in the amide I and amide III regions. We also noted that the average spectral information from each cluster was not well defined and showed mostly a differentiation in fat concentrations and structures with protein bands not being well defined in some spectral regions, which made it difficult to elucidate the secondary protein structures in the amide regions of the spectra.

Two alternative methods to $\mathrm{K}$-means clustering were also applied to the image data: MCR-ALS and NMF. Unsupervised MCR-ALS is commonly used to analyse spectral image data; in this case, it proved to differentiate the three components of interest in the cheese matrix easily by applying a minimum of three end members to the algorithm. ${ }^{16,17}$ Although smaller fat globules appear to be contained within the casein component map instead of the fat component map, as in Figure 8, this could possibly be resolved by further chemometric techniques that were not investigated in this study. Examination of the spectra show the complete absence of water signals in the region $3100-3550 \mathrm{~cm}^{-1}$ in the fat (red) spectra along with indicative carbonyl signal from the fat triglyceride at $1740 \mathrm{~cm}^{-1}$. The whey and casein spectra are distinguished, as indicated in Figures 4B and 5. MCR-ALS methods have the additional benefit of estimating the concentrations of any of the defined components in any pixel of an image and are useful for interrogating the proportionate mix of the resulting spectral image data, and we suggest they are worth further investigation with more advanced constrained MCR-ALS methods in the future. The third multivariate method applied was unsupervised NMF, which allowed a constraint of nonnegativity to be applied in the algorithm. Non-negativity 

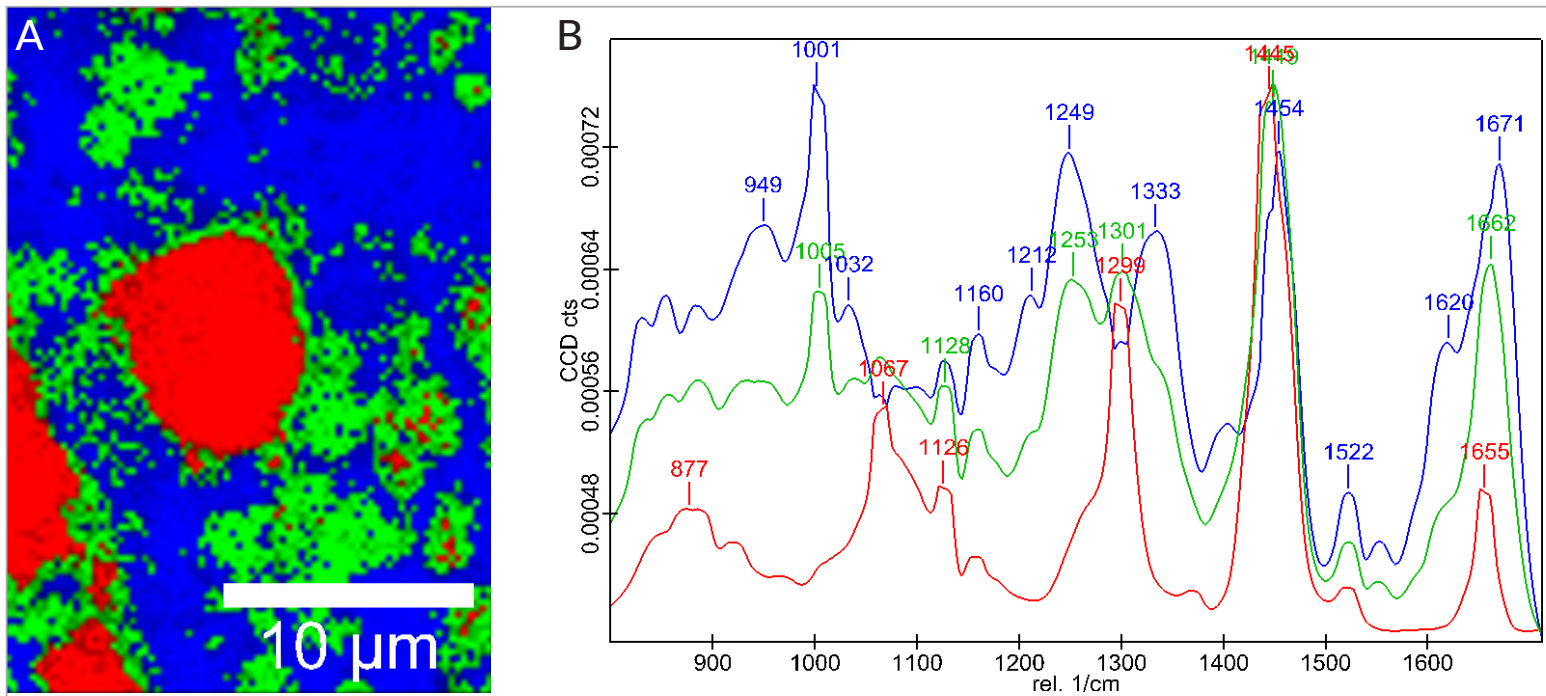

Figure 9. NMF map and associated spectral data of an ROI in the whey protein particle sample: whey protein = green; fat $=$ red; casein $=$ blue .

allows the data components to more fully explain the spectral variation at each pixel.

The NMF algorithm appears to give more defined spectral data from the resulting components than K-means clustering: it allowed better insights from analysing the differences between spectra generated from each final component, as seen in Figure 9. However, we note that it took five components to extract the whey protein particles as opposed to three components with the MCR-ALS method. NMF used fewer components than the K-means clustering method; it also afforded better information on the spectral differences between mapped components. This allowed imaging of the whey protein particles, as well as of the whey protein and casein, illustrating their distribution from the differentiation in their amide bond structures and aromatic side chain composition; also of interest is the distribution of whey protein around the interface of the fat globules.

In general, we found that applying unsupervised methods led to slight variations in the results, depending on the image or ROI analysed. Both K-means clustering and NMF took more end members to extract the whey protein particles, with NMF extracting some major differences in fat fractions before the differences within any protein components. In contrast, K-means clustering appeared to extract differences around the interface of the fat and casein protein phase before the differences between whey protein and casein with less differentiation in the spectral data. NMF gave satisfactory spectral and structural differentiation and allowed interrogation of the spectral data efficiently, whereas the average spectral information using K-means proved spectra to be poorly differentiated when comparing differences between average cluster spectra for the various algorithms. In contrast, MCR-ALS gave good spectral data but on occasion generated very noisy average spectra, so we considered it not as reliable for interpreting spectral differences. While we would note that the changes in the spectral data from fat appear to be more pronounced than those from the protein in this model cheese sample, in cheese production and use differences in protein distribution can have major implications and is our focus in this research. The ability to use a combination of peak mapping, K-means and NMF clustering approaches allows the differentiation of subtle spectral differences between whey and casein and also suggests that with Raman microscopy we can understand protein secondary structure variation that may contribute to cheese attributes.

\section{Conclusions}

The results showed that Raman confocal imaging techniques could readily differentiate the whey protein particles in a model cheese matrix composed of casein and fat by either peak interval mapping or multivariate clustering methods. Unconstrained MCR-ALS gave satisfactory image reconstruction of the sample, but in-depth analysis of the associated spectral data and detailed interrogation 
of the image illustrated that the distribution of components was not well defined. NMF gave better results, with more defined spectral information for mapped components. In particular, the amide I and amide III bands illustrated that the whey protein particles were rich in $\beta$-sheet structures, in comparison with the casein matrix in the model cheese. Overall, of the multivariate methods, NMF was found to be superior, as it better distinguished clusters in the complex cheese matrix. The use of a range of approaches has benefits in terms of an ability to apply Raman imaging to match chemical distribution to structure. The further investigation of compositionally well-understood cheese systems to strengthen this link will be a focus of our future research. We note that, because of the variability in the results depending on the algorithm used, we suggest that a thorough investigation of a range of image mapping algorithms and their application is required to gain insights from the Raman image data of complex systems such as cheese.

\section{References}

1. T. Lei and D.W. Sun, "Developments of nondestructive techniques for evaluating quality attributes of cheeses: a review", Trends Food Sci. Technol. 88, 527-542 (2019). https://doi.org/10.1016/j. tifs.2019.04.013

2. H. Fischer and A. Jauss, "Food analysis with confocal Raman microscopy", Spectroscopy 29 (2007).

3. E.C.Y. Li-Chan, "The applications of Raman spectroscopy in food science", Trends Food Sci. Technol. 71(11), 361-370 (1996). https://doi.org/10.1016/ S0924-2244(96)10037-6

4. E.C.Y. Li-Chan, "Vibrational spectroscopy applied to the study of milk proteins", Lait 87(4-5), 443-458 (2007). https://lait.dairy-journal.org/articles/lait/ abs/2007/04/107s4516/I07s4516.html

5. A.M. Herrero, "Raman spectroscopy for monitoring protein structure in muscle food systems", Crit. Rev. Food Sci. Nutr. 48(6), 512-523 (2008). https://doi. org/10.1080/10408390701537385

6. M. Motoyama, M. Ando, K. Sasaki, I. Nakajima, K. Chikuni, K. Aikawa and H. Hamaguchi, "Simultaneous imaging of fat crystallinity and crystal polymorphic types by Raman microspectroscopy", Food Chem. 196, 411-417 (2016). https://doi. org/10.1016/j.foodchem.2015.09.043
7. G. Xiong, M. Han, Z. Kang, Y. Zhao, X. Xu and $Y$. Zhu, "Evaluation of protein structural changes and water mobility in chicken liver paste batters prepared with plant oil substituting pork back-fat combined with pre-emulsification", Food Chem. 196, 388-395 (2016). https://doi.org/10.1016/j.foodchem.2015.09.068

8. E. Li-Chan and S. Nakai, "Importance of food proteins in food emulsions", in Microemulsions and Emulsions in Foods. ACS Symposium Series Vol. 448, Ed by M. El-Nokaly and D. Cornell. ACS Publications, Washington, DC, USA, Ch. 15, pp. 193-212 (1991). https://doi.org/10.1021/bk-1991-0448.ch015

9. N. Linlaud, E. Ferrer, M.C. Puppo and C. Ferreo, "Hydrocolloid interaction with water, protein, and starch in wheat dough", J. Agric. Food Chem. 59(2), 713-719 (2011). https://doi.org/10.1021/jf1026197

10. Z.-L. Kang, P. Wang, X.-L. Xu, C.-Z. Zhu, Y.-F. Zou, K. Li and G.-H. Zhou, "Effect of a beating process, as a means of reducing salt content in Chinesestyle meatballs (kung-wan): a dynamic rheological and Raman spectroscopy study", Meat Sci. 96(2), 669-674 (2014). https://doi.org/10.1016/j. meatsci.2013.09.024

11. M. Bouraoui, S. Nakai and E. Li-Chan, "In situ investigation of protein structure in Pacific whiting surimi and gels using Raman spectroscopy", Food Res. Int. 30(1), 65-72 (1997). https://doi.org/10.1016/S09639969(97)00020-3

12. E. Li-Chan, S. Nakai and M. Hirotsuka, "Raman spectroscopy as a probe of protein structure in food systems", in Protein Structure-Function Relationships in Foods, Ed by R.Y. Yada, R.L. Jackman and J.L. Smith. Springer, Boston, MA, Ch. 8, pp. 163 (1994). https:// doi.org/10.1007/978-1-4615-2670-4_8

13. S. Ngarize, H. Herman, A. Adams and N. Howell, "Comparison of changes in the secondary structure of unheated, heated, and high-pressure-treated $\beta$-lactoglobulin and ovalbumin proteins using Fourier transform Raman spectroscopy and self-deconvolution", J. Agric. Food Chem. 52(21), 6470-6477 (2004). https://doi.org/10.1021/jf030649y

14. G.P.S. Smith, S.E. Holroyd, D.C.W. Reid and K.C. Gordon, "Raman imaging processed cheese and its components", J. Raman Spectrosc. 48(3), 374-383 (2017). https://doi.org/10.1002/jrs.5054

15. H. Mitsutake, R.J. Poppi and M.C. Breitkreitz, "Raman imaging spectroscopy: history, fundamentals 
and current scenario of the technique", J. Braz.

Chem. Soc. 30(11), 2243-2258 (2019). https://doi.

org/10.21577/0103-5053.20190116

16. G. van Dalen, E.J.J. van Velzen, P.C.M. Heussen, M. Sovago, K.F. van Malssen and J.P.M. van Duynhoven, "Raman hyperspectral imaging and analysis of fat spreads", J. Raman Spectrosc. 48(8), 1075-1084 (2017). https://doi.org/10.1002/jrs.5171

17. B. Prats-Mateu, M. Felhofer, A. de Juan and N. Gierlinger, "Multivariate unmixing approaches on Raman images of plant cell walls: new insights or overinterpretation of results?", Plant Methods 14, 52 (2018). https://doi.org/10.1186/s13007-018-0320-9 